\title{
Lempel-Ziv complexity analysis of local field potentials in different vigilance states with different coarse-graining techniques
}

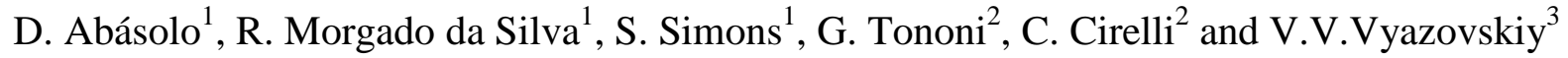 \\ ${ }^{1}$ Centre for Biomedical Engineering, Department of Mechanical Engineering Sciences, Faculty of Engineering and Physical Sciences, \\ University of Surrey, Guildford, UK \\ ${ }^{2}$ Department of Psychiatry, University of Wisconsin-Madison, Madison, Wisconsin, United States of America \\ ${ }^{3}$ Department of Biochemistry and Physiology, Faculty of Health and Medical Sciences, University of Surrey, UK
}

\begin{abstract}
Analysis of electrophysiological signals recorded from the brain with Lempel-Ziv (LZ) complexity, a measure based on coarse-graining of the signal, can provide valuable insights into understanding brain activity. LZ complexity of local field potential signals recorded from the neocortex of 11 adult male Wistar-Kyoto rats in different vigilance states waking, non-rapid-eye movement (NREM) and REM sleep was estimated with different coarse-graining techniques (median, LZCm, and k-means, LZCkm). Furthermore, surrogate data were used to test the hypothesis that LZ complexity results reveal effects accounted for by temporal structure of the signal, rather than merely its frequency content. LZ complexity values were significantly lower in NREM sleep as compared to waking and REM sleep, for both real and surrogate signals. LZCkm and LZCm values were similar, although in NREM sleep the values deviated in some epochs, where signals also differed significantly in terms of temporal structure and spectral content. Thus, the interpretation of $\mathrm{LZ}$ complexity results should take into account the specific algorithm used to coarsegrain the signal. Moreover, the occurrence of high amplitude slow waves during NREM sleep determines $L Z$ complexity to a large extent, but characteristics such as the temporal sequence of slow waves or cross-frequency interactions might also play a role.
\end{abstract}

Keywords - Lempel-Ziv complexity, k-means, local field potential, surrogate data, sleep

\section{INTRODUCTION}

Different brain states are associated with changes in neuronal network function and characterized by specific spatiotemporal patterns of cortical activity [1]. Three main vigilance states are usually identified in mammals including humans: waking, non-rapid-eye movement (NREM) and REM sleep. They are distinguished not only based on animal's behavior but also based on the total amplitude and spectral power of cortical electroencephalogram (EEG) and local field potential (LFP) [2]. The functional significance of the difference in brain activity between vigilance states is not yet understood.

While much has been learned about different brain states using conventional spectral analysis, non-linear analysis metrics are still rarely used. This becomes especially relevant when signals differ drastically in their frequency content and amplitude.

Non-linear analysis of EEG or LFP signals with LempelZiv (LZ) complexity, a measure of complexity based on coarse-graining of the signal, can provide valuable insights into understanding brain activity [3], [4]. LZ complexity is a method of symbolic sequence analysis and, as such, is based on a coarse-graining of the time series being investigated. The signal must be transformed into a finite symbol sequence before estimating its LZ complexity. While the choice of coarse-graining approaches may appear essential for interpretability of LZ complexity values obtained in different vigilance states, no attempts to perform a comparison between coarse-graining techniques have been made. In this study, we applied LZ complexity analysis to cortical LFP recordings collected in freely-moving rats during spontaneous waking and sleep states with different coarsegraining techniques. Furthermore, surrogate data were used to test the hypothesis that LZ complexity results reveal effects purely accounted for by temporal structure, rather than frequency content, and may provide additional insights into the characteristics of the signal that contribute to the information content.

This paper is organized as follows. In section 2 we introduce the LFP signals, the different coarse-graining methods applied, the surrogate data used, and the statistical evaluations carried out. Results are presented in section 3 and our findings are discussed in section 4 , where the conclusions of the study are also presented.

\section{MAterial AND METHODS}

\section{A. Materials}

LFP recordings and single- and multi-neuron activity were collected with microwire arrays implanted in the frontal cortex of 11 adult male Wistar-Kyoto rats during undisturbed waking and sleep [2]. Three stages - waking, NREM and REM sleep - were identified offline based on 
the EEG/LFP signals and electromyogram (EMG), as well as frame-by-frame analysis of the video recording. For this analysis one period of stable continuous awake state has been chosen, along with one individual episode of NREM and REM sleep in each rat.

The total amount of vigilance states that contributed to these analyses was $8.5 \pm 0.6,12.2 \pm 2.3$ and $3.0 \pm 0.2$ minutes for waking (W), NREM sleep (N) and REM sleep $(\mathrm{R})$, respectively. Examples of the three different vigilance states (8-s epochs) are shown in Figure 1.

\section{Waking}

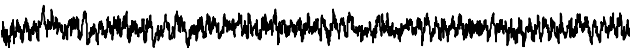

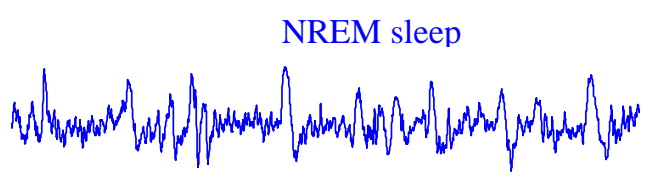

REM sleep

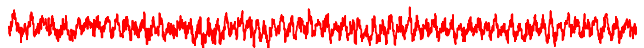

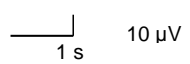

Fig. 1 Representative 8-s LFP traces recorded from the frontal cortex in a freely-behaving rat in spontaneous waking, NREM and REM sleep.

\section{B. Lempel-Ziv complexity}

The LZ algorithm [5] is a method of symbolic sequence analysis that can be used to measure the complexity of finite length sequences. Several aspects of this method make it attractive for biomedical signal processing: it is nonparametric, it can be applied to estimate the complexity of relatively short sequences, and it does not assume a particular model (e.g. chaotic or stochastic) for the system generating the time series [6]. In addition, the computational cost of estimating LZ complexity is significantly lower than that of embedding entropy methods in widespread use, such as approximate or sample entropy. This is a considerable advantage for the analysis of chronic sleep recordings.

LZ complexity is based on the coarse-graining or symbolization of the original time series. This process involves converting the original biomedical signal into a sequence with a finite number of symbols. In most cases, a binary conversion is used. The coarse-graining process determines how much information can be retained from the original signal. In this paper, two different solutions - using the median as the threshold due to its robustness to outliers [7] or k-means in the symbolization of the original signal [8] are presented and compared.

a) Median. The signal is converted into a binary sequence $P=s(1), s(2), \ldots, s(n)$ by comparing each sample $x(i)$ with a threshold, in this case the median of the time series $T_{d}$, with $s(i)$ then given by [6]:

$$
s(i)= \begin{cases}0 & \text { if } x(i)<T_{d} \\ 1 & \text { if } x(i) \geq T_{d}\end{cases}
$$

b) k-means. This approach is based on the grouping of data around centroids corresponding to points around which most of the data is agglomerated [8]. The number of centroids $k$ is defined by the user and is equal to 2 for binary sequences.

In the initial iteration of the method, one must set the two initial centroids as follows:

$$
\begin{aligned}
& z_{1}(1)=x_{m}+\varepsilon \cdot x_{m} \\
& z_{2}(1)=x_{m}-\varepsilon \cdot x_{m}
\end{aligned}
$$

where we assume $\varepsilon=0.005$ and $x_{m}$ is the mean of the data points from the original signal, $x(i)$ [8]. Distances of each data point to centroids are then calculated as:

$$
\begin{aligned}
& D_{1}^{i}=\left\|x(i)-z_{1}(1)\right\|^{2} \\
& D_{2}^{i}=\left\|x(i)-z_{2}(1)\right\|^{2}
\end{aligned}
$$

The signal can then be converted into a binary sequence $P=s(1), s(2), \ldots, s(n)$ with $s(i)$ given by the following equation [8]:

$$
s(i)= \begin{cases}1 & \text { if } D_{1}^{i}<D_{2}^{i} \\ 0 & \text { if } D_{1}^{i} \geq D_{2}^{i}\end{cases}
$$

Thus, each data point is set based on a minimum distance criterion. All points assigned with symbol 1 will belong to group 1, and points assigned with symbol 0 will belong to group 2 . In a new iteration, two new centroids have to be defined. For group $1, z_{1}(2)$ is the average coordinate among all the members in the group. For group 2, $z_{2}(2)$ is the average coordinate among all members of the group. Equations (4) and (5) are then re-applied in order to find the new distance values and the new symbolic sequence $P$. The procedure has to be repeated until $z_{1}(j+1)=z_{2}(j)$ for all $j$.

Once the symbolic sequence $P$ has been created, its complexity needs to be estimated. This has been done using the parsing process suggested by Lempel and Ziv [5], which scans $P$ from left to right and adds one unit to a complexity 
counter $c(n)$ every time a new subsequence of consecutive characters is found. In this way, LZ complexity is related to the number of distinct substrings and the rate of their recurrence along the given sequence [9]. As a result, LZ complexity is very much in the spirit of Kolmogorov's algorithmic complexity [10], [11]. A detailed description of the complexity algorithm can be found in [6].

Last, but not least, the complexity counter has to be normalized to obtain a complexity measure independent of the sequence length. In general, the upper bound of the complexity is given by [5]:

$$
b(n) \equiv \frac{n}{\log _{\alpha}(n)}
$$

$\alpha$ is the number of symbols in the alphabet (hence $\alpha=2$ for a binary conversion). Therefore, the normalized LZ complexity can be defined as:

$$
C(n)=\frac{c(n)}{b(n)}
$$

\section{Surrogate data}

To investigate whether the complexity of signals derived from brain activity is not merely an artifact of the spectral frequency content, surrogate signals were generated from the LFP signals. Firstly, the Fourier transform of the signals was computed and the phases of the Fourier coefficients randomized while at the same time keeping unchanged their magnitude [12]. Then, the inverse Fourier transform into the time domain was performed. As a result, surrogates indistinguishable from naturalistic LFP signals with respect to spectral characteristics were obtained.

\section{Statistical analysis}

The LZ complexity values are reported as means \pm standard error of the mean (SEM). Two-tailed paired t-tests were used to evaluate significant differences between LZ complexity values obtained in different vigilance states and between different coarse-graining techniques within a state.

\section{RESULTS}

LZ complexity was computed for the LFP signals with the two coarse-graining methods aforementioned, k-means (LZCkm) and median (LZCm), over consecutive 4-s epochs. We found that average LZ complexity values, computed over consecutive 4-s epochs, were invariably substantially lower in NREM sleep as compared to waking and REM sleep (Figure 2).
We next used two different methods of coarse-graining the signals to compare if vigilance-state specific differences are affected by the algorithm. This appeared not to be the case, as the values were virtually identical between k-means (LZCkm) and median (LZCm) coarse-graining approaches (results are summarized in Table 1). The differences between results obtained with $\mathrm{LZCkm}$ and $\mathrm{LZCm}$ were not statistically significant.

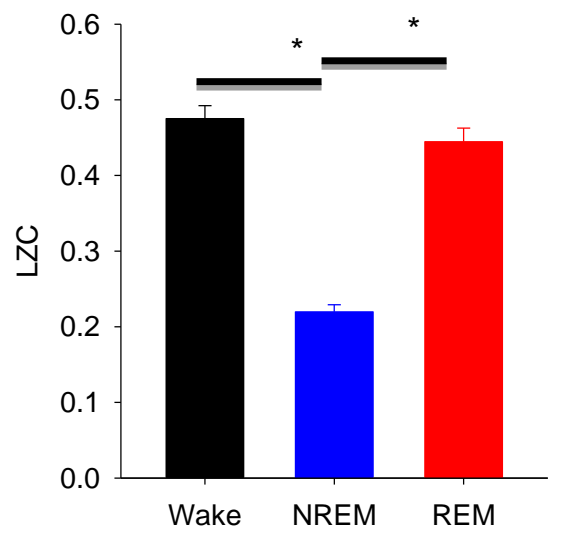

Fig. 2. Average LZ complexity (LZC) values in the three behavioral states $(\mathrm{n}=11$ rats). The values are obtained with $\mathrm{k}$-means approach. Asterisks above the bars depict significant differences $(p<0.05)$

Table 1 LZ complexity results for waking (W), NREM sleep (N) and REM sleep (R) obtained with k-means (LZCkm) and median (LZCm) coarse-graining approaches

\begin{tabular}{cccc}
\hline Method & W & N & R \\
\hline LZCkm & $0.46 \pm 0.046$ & $0.25 \pm 0.031$ & $0.47 \pm 0.027$ \\
LZCm & $0.46 \pm 0.046$ & $0.25 \pm 0.030$ & $0.47 \pm 0.026$ \\
\hline
\end{tabular}

However, while on average LZCkm and LZCm values were similar, during a subset of 4-s epochs the values deviated, and that was especially apparent in NREM sleep. On average, the proportion of epochs where absolute difference (either positive or negative) between LZCkm and LZCm values exceeded $10 \%$ was $2.7 \pm 2.4 \%, 16.8 \pm 4.3 \%$ and 1.3 $\pm 1.2 \%$ of all 4 -s epochs in waking, NREM and REM sleep respectively. Interestingly, in NREM sleep, where the proportion of epochs where LZCkm and LZCm values differed by more than $10 \%$ was substantial, signal variance and LFP power in slow-wave range showed a systematic difference as well. Specifically, the difference in both signal variance and slow wave activity (SWA) showed higher values during those epochs where LZCkm and LZCm were different (variance: $81.8 \pm 16.9$ vs. $58.3 \pm 13.9 \mu \mathrm{V}, p=0.0351$, paired ttest; relative SWA: $121.5 \pm 2.6$ vs. $95.1 \pm 1.5 \%$ of mean SWA over all epochs, $p=7.3030 \mathrm{e}-004$, paired t-test).

LZ complexity values were significantly lower in NREM sleep as compared to waking and REM sleep for both real 
and surrogate signals. Interestingly, average LZ complexity values for surrogate signals differed only marginally for waking (increase by $1.3 \%$ ) and REM sleep (decrease by $1.4 \%$ ), but were significantly lower in NREM sleep (decrease by $8.4 \pm 1.4 \%$ ). Computing the distribution of LZ complexity values revealed that this difference is accounted by fewer epochs with higher LZ complexity values for the surrogate data than for the real data.

\section{DisCUSSION AND CONCLUSIONS}

In this study we performed a detailed analysis of LZ complexity derived from cortical LFP signals in freely behaving rats. Since the patterns of spontaneous cortical activity are different between different vigilance states [2] we hypothesized that the information content of the signals is state-dependent. As expected, we found that the values of LZ complexity were substantially lower in NREM sleep, which is considered a state functionally disconnected from the environmental input, and is characterized by absent or reduced consciousness [13].

We also found that different approaches of coarsegraining have only marginal overall influence on the resulting values of LZ complexity in waking and REM sleep, while the differences in NREM sleep were more pronounced. Interestingly, in those epochs where there was an effect of coarse-graining technique, signals also differed significantly in terms of temporal structure and spectral content. Thus, interpreting the information measures obtained with LZ complexity should take into account the specific algorithm used to coarse-grain the signal. Our analyses also showed vigilance state-specific differences and similarities between LZ complexity values computed from real and surrogate data. The data suggest that the occurrence of high amplitude slow waves during NREM sleep determines LZ complexity to a large extent, but other characteristics, such as the temporal sequence of slow waves or cross-frequency interactions might also play a role.

An important implication of our results is that LZ complexity may not only provide information above and beyond of what can be obtained with conventional power spectral analysis, but, in fact, be the only option for studies in which signals to be compared are inherently different, such as due to a difference in age, gender, ethnic origin, pharmacological treatment, etc. Therefore, introducing LZ complexity as a new tool to investigate brain signals in waking and sleep is important. On one hand, using non-linear analysis can provide sensitive measures, relatively independent from spectral power. On the other hand, LZ complexity values can provide unique insights into the network mechanisms of waking and sleep, and pave the way towards gaining better understanding of their physiological and functional relevance.

Although results are promising, the sample size was small. As a result, our findings are preliminary and require replication in a larger database before any conclusion can be made of its potential impact.

\section{ACKNOWLEDGMENT}

This work was supported by the Engineering and Physical Sciences Research Council [grant number EP/I000992/1].

\section{REFERENCES}

1. Buzsáki, G (2006) Rhythms of the brain. Oxford University Press, Oxford ; New York, 2006.

2. Vyazovskiy VV, Olcese U, Lazimy YM et al. (2009) Cortical firing and sleep homeostasis. Neuron 63:865-878 DOI 10.1016/j.neuron.2009.08.024

3. Abásolo D, Homero R, Gómez C et al. (2006) Analysis of EEG background activity in Alzheimer's disease patients with Lempel-Ziv complexity and central tendency measure, Med Eng Phys 28:315-322 DOI 10.1016/j.medengphy.2005.07.004

4. Arnold MM, Szczepanski J, Montejo N et al. (2012) Information content in cortical spike trains during brain state transitions. J Sleep Res: 22:13-21 DOI 10.1111/j.1365-2869.2012.01031.x

5. Lempel A, Ziv J (1976) On the complexity of finite sequences. IEEE Trans Inform Theory 22:75-81 DOI 10.1109/TIT.1976.1055501

6. Zhang XS, Roy RJ, Jensen EW (2001) EEG complexity as a measure of depth of anesthesia for patients. IEEE Trans Biomed Eng 48:14241433 DOI 10.1109/10.966601

7. Nagarajan R (2002) Quantifying physiological data with Lempel-Ziv complexity - Certain issues. IEEE Trans Biomed Eng 49:1371-1373 DOI 10.1109/TBME.2002.804582

8. Zhou S, Zhang Z, Gu J (2011) Interpretation of coarse-graining of Lempel-Ziv complexity measure in ECG signal analysis, Proc. 33rd Annual Conference of the IEEE EMBS, Boston, USA, pp. 2716-2719

9. Radhakrishnan N, Gangadhar BN (1998) Estimating regularity in epileptic seizure time-series data. A complexity-measure approach. IEEE Eng Med Biol 17:89-94 DOI 10.1109/51.677174

10. Kolmogorov AN (1965) Three approaches to the quantitative definition of information. Infor Trans 1:3-11.

11. Hu J, Gao J, Principe JC (2006) Analysis of biomedical signals by the Lempel-Ziv complexity: the effect of finite data size. IEEE Trans Biomed Eng 53:2606-2609 DOI 10.1109/TBME.2006.883825

12. Palus M, Hoyer D (1998) Detecting nonlinearity and phase synchronization with surrogate data. IEEE Eng Med Biol Mag 17:40-45 DOI $10.1109 / 51.731319$

13. Tononi, G (2012) Integrated information theory of consciousness: an updated account. Arch Ital Biol 150:56-90 DOI 10.4449/aib.v149i5.1388

Author: Daniel Abásolo

Institute: University of Surrey

City: Guildford

Country: United Kingdom

Email: d.abasolo@surrey.ac.uk 